\title{
Social Media and Scientific Meetings: An Analysis of Twitter Use at the Annual Meeting of the American Society of Neuroradiology
}

\author{
(D) A. Radmanesh and A.L. Kotsenas
}

G iven the enormous potential and easy access by using mobile devices, social media are being increasingly used during radiology and other medical specialty meetings (Figure). ${ }^{1-5}$ Social media use during scientific meetings allows attendees to post commentaries on sessions, questions about conference logistics, calls for casual meetings ("tweet-ups"), or even tips for a good dinner. It also allows meeting organizers to make announcements and nonattendees to participate in the conference virtually.

Twitter users can embed metadata "tags" in their posts to make them searchable by using hashtags, a word or acronym preceded by the \# character. Given the 140-character limit for each tweet, brief or abbreviated hashtags are usually favored. Many specialty meeting organizers now publish a meeting-specific hashtag such as \#ASNR14 used at the 52nd annual meeting of the American Society of Neuroradiology (ASNR).

In many professional meetings currently, hallways and foyers are equipped with large screens displaying all meeting-related tweets posted from inside or outside the venue. These make it easy for attendees to have a glance at the latest posts during scheduled breaks or as they walk from one session to another. In large meetings where many tweets are posted, each session may be assigned a specific hashtag so that people can follow the streams related to their session of interest and post questions to presenters and moderators. Users can also "reply" to others' posts to start a conversation on the topic. People who share interests can "follow" each other's posts, arrange to meet in person, and stay in touch even after the conference is over. Sometimes, these professional connections last for years.

Recognizing the current pattern of social media use in our subspecialty society can guide planning for future societal meetings to take advantage of the existing potentials. In this article, we will analyze Twitter use during the 2014 annual meeting of the American Society of Neuroradiology (May 17-22, 2014, Montréal, Quebec, Canada).

From the Department of Radiology and Biomedical Imaging (A.R.), University of California, San Francisco, San Francisco, California; and Department of Radiology (A.L.K.), Mayo Clinic, Rochester, Minnesota.

Please address correspondence to Alireza Radmanesh, MD, Neuroradiology Section, UCSF, 505 Parnassus Ave, L-352, San Francisco, CA 94143; e-mail: Alireza.Radmanesh@ucsf.edu; @AliRadmanMD

http://dx.doi.org/10.3174/ajnr.A4168
We reviewed all Twitter posts (Twitter.com, San Francisco, California) that included the meeting hashtag \#ASNR14 and were posted from May 7, 2014 (the date the hashtag was registered with Symplur) to May 22, 2014 (midnight following the final day of the conference). The transcripts of the tweets were obtained from Symplur (Symplur, Upland, California; Symplur.com), a health care social media analytics organization.

The number of participants (microbloggers) and the number of tweets posted by each were recorded. On the basis of the information on Twitter account profiles, the microbloggers were categorized into radiologists, nonradiologist physicians/postdoctoral researchers, radiology technologists, nurses, vendors, social media professionals, journals, imaging societies, and the host city. The content of each tweet was categorized into commentary on meeting sessions, questions directed to presenters/moderators, meeting-related announcements, questions about meeting logistics, commentary about the use of social media for health care/ meeting-related purposes, arranging tweet-ups, status updates, journal promotions, vendor marketing promotions, and not otherwise categorized. We analyzed the original tweets and those that were reposted by other users as "retweets" or "favorites." For our analysis, retweets and favorites were grouped together. The language in which tweets were posted was also recorded. Analysis was performed by using the statistical tools of Excel (Microsoft, Redmond, Washington).

Fifty-four microbloggers posted 410 tweets with the \#ASNR14 hashtag during May 7-22, 2014. The breakdown of the microbloggers can be seen in Table 1 . Of 410 total tweets, 238 tweets $(68 \%)$ were original posts, and the rest were retweets or favorites. The posted tweets resulted in 415,102 total views or impressions. Nine tweets $(2.2 \%)$ were posted in Spanish. A few posts contained phrases in French, the official language of the host city, but none were posted predominantly using that language. The remainder of the tweets were posted in English.

A mean of 8 tweets per participant was generated (range, 1-119; SD, 19; median, 2). The top 3 tweeters, all neuroradiologists, generated 223 (54.4\% of all) tweets.

The most common tweet content was related to commentary on sessions, which encompassed 202 (49\% of all) tweets. Social tweets, including those related to the use of social media, arranging tweet-ups, and participant status updates accounted for 176 


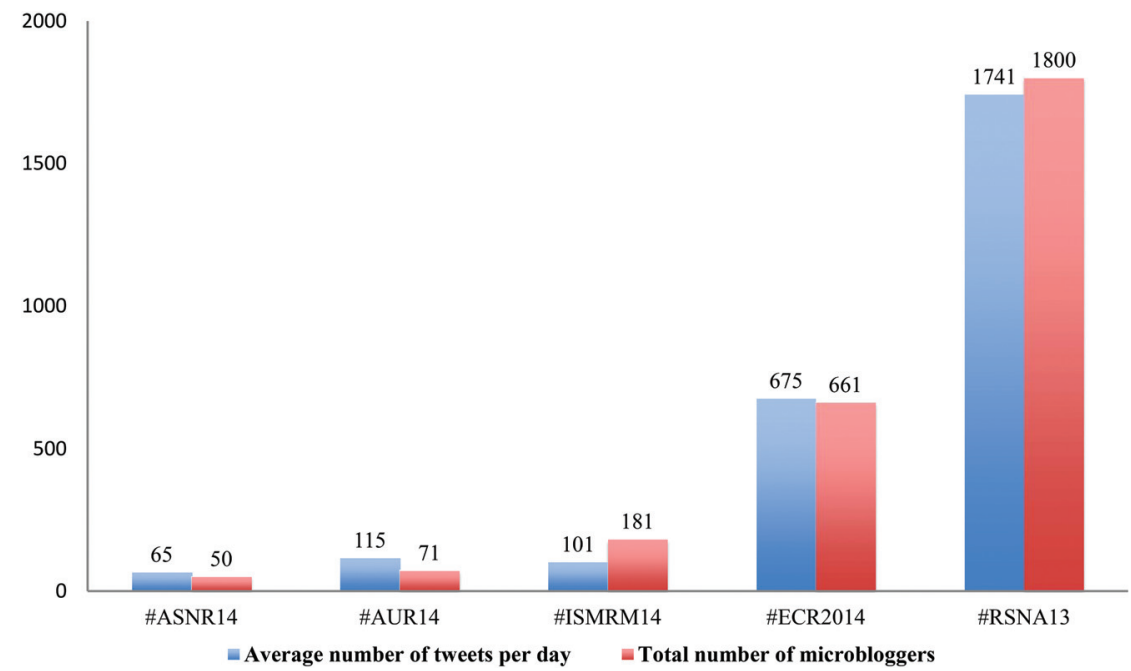

FIG. Average number of tweets per day and total number of microbloggers for the duration of conferences at some most recent societal conferences in radiology. The difference in the number of tweets or microbloggers among different radiology societal meetings is at least in part related to the difference in the number of attendees. Of note, some of the tweets related to each meeting were posted by microbloggers who were not attending the venue. ASNR14 indicates American Society of Neuroradiology, May 17-22, 2014, Montréal, Quebec, Canada; AUR14, Association of University Radiologists, April 1-4, 2014, Baltimore, Maryland; ISMRM14, International Society for Magnetic Resonance in Medicine, May 10-16, 2014, Milan, Italy; ECR2014, European Congress of Radiology, March 6-10, 2014, Vienna, Austria; RSNA13, Radiological Society of North America, November 30-December 5, 2013, Chicago, Illinois. Data were obtained from Symplur.com.

Table 1: Analysis of microbloggers posting tweets with \#ASNR14 hashtag during May 7-22, 2014

\begin{tabular}{lc}
\hline \multicolumn{1}{c}{ Microblogger Category } & $\begin{array}{c}\text { No. (\% of All } \\
\text { Microbloggers) }\end{array}$ \\
\hline Radiologist & $20(37.0 \%)$ \\
Vendors & $11(20.1 \%)$ \\
Radiology/imaging departments & $7(13 \%)$ \\
Other physicians/postdoctoral researchers & $5(9.3 \%)$ \\
Social media professionals & $4(7.4 \%)$ \\
Radiology journals & $3(5.6 \%)$ \\
Technologists & $1(1.9 \%)$ \\
Nurses & $1(1.9 \%)$ \\
Host city & $1(1.9 \%)$ \\
Unidentifiable & $1(1.9 \%)$ \\
Total & 54 \\
\hline
\end{tabular}

Table 2: Analysis of content for tweets with \#ASNR14 hashtag posted during May 7-22, 2014

\begin{tabular}{lccc}
\hline \multicolumn{1}{c}{ Content Category } & $\begin{array}{c}\text { Total No. } \\
\text { (\% of Total } \\
\text { Tweets) }\end{array}$ & $\begin{array}{c}\text { Original Posts } \\
\text { (\% of Total } \\
\text { Original Tweets) }\end{array}$ & $\begin{array}{c}\text { Reposts } \\
\text { (\% of Original } \\
\text { Posts in }\end{array}$ \\
\hline That Category)
\end{tabular}

(42.9\% of all) tweets. See Table 2 for a complete breakdown of tweet content. No questions were posted to the presenters or moderators.
The session that generated the greatest number of tweets was "The Foundation of the ASNR Special Session on Traumatic Brain Injury: Is DTI Ready for Prime Time?" (44 tweets accounting for $21.8 \%$ of the tweets related to session content) followed by Dr Stanely Prusiner's keynote address "A Unifying Role for Prions in Neurodegenerative Diseases" (13 tweets accounting for 6.4\% of the tweets related to session content).

Social tweets were most likely to be retweeted (84 of 176 tweets in that category, $47.7 \%$ ), followed by tweets related to session content (78 of 202 tweets in that category, 49.2\%). Vendor marketing tweets were least likely to be retweeted ( 1 of 9 tweets, $11.1 \%$ ).

Our analysis shows that Twitter use by radiologists at the annual meeting of the ASNR is still in its infancy. Given that there were only 20 radiologist microbloggers using the hashtag \#ASNR14, it is clear that the neuroradiology community has not yet fully embraced the use of social media for this purpose. This lack of participation likely contributes to the paucity of content related to meeting logistics and questions directed to meeting organizers. This scenario may be because of insufficient knowledge of the potentials for professional use of social media at medical conferences and scientific meetings. Although the @ASNRStaff Twitter handle has indeed been inactive, organizers of the ASNR meeting have attempted to promote Twitter use at each of the prior two meetings by building a Twitter feature into the meeting Guidebook mobile application.

To our knowledge, our analysis is the first to look at specific meeting-related tweet content for a radiology meeting. Most of the posts at the annual meeting were related to session content. Of particular interest was the debate session on the use of diffusion tensor imaging for traumatic brain injury, which prompted comments by many postdoctoral researchers and members of the neuroimaging community who virtually participated in the discussion. Not surprisingly, this was followed by social content relating to the use of social media for meeting-related purposes, planning tweet-ups with other users, and alerting followers to the current "status" of the user or his or her presence at the meeting. Vendor marketing content made up a distinct minority of posts and was least likely to be retweeted or marked as favorite by microbloggers.

Although some have argued that live-tweeting lectures is a form of "neoliberalism" and is more an attempt at personal branding than at scholarship, " "live-tweeting encouraged" has become the default mode for many scientific meetings. Presenters are encouraged to share their Twitter handles with the audience during opening remarks so that the handle can be used to quote, paraphrase, or discuss the work. Some meeting organizers have established optional inclusion of Twitter handles on attendee identification badges. 
Social media channels open up convention floors to members and scholars from around the world who have been unable to physically attend, while rendering attendees' academic accomplishments more visible to the public. ${ }^{7}$ In a study of social media participation at an international emergency medicine conference, more than $60 \%$ of individuals posting tweets were not physically present at the meeting. ${ }^{3}$ During the 2014 ASNR meeting, approximately $50 \%$ of radiologists posting with the \#ASNR14 hashtag attended the meeting virtually. ${ }^{8}$

Some attendees have found live-tweeting to be a great way to take notes and remain focused on what is being presented, ${ }^{9}$ while others may find it a source of distraction. As more and more conferences adopt a parallel-session format, social media users can virtually attend more than one session at the same time by reading the posts from other sessions, either as they come in or-if distraction is a concern-later by searching the posts or reviewing a transcript created by an enabler such as Symplur.

During the 2014 meeting of the International Society for Magnetic Resonance in Medicine (\#ISMRM14), audience members were given the opportunity to pose their questions to the presenters and moderators by using session-specific hashtags, and moderators were instructed to monitor the session-specific feeds for questions and comments (C.P. Hess, $\mathrm{MD}, \mathrm{PhD}$, personal e-mail communication, July 18, 2014). This option can be explored at future ASNR meetings.

Social media platforms are considered public domains. Some presenters or panelists may feel uncomfortable about having their findings mentioned on social media before having them published as a journal article. In such cases, presenters or panelists are strongly encouraged to make an announcement to that effect to the audience at the beginning of the presentation. In the near future, organizers of scientific meetings may ask presenters to reveal their wishes in regard to social media coverage at the time of submission or final acceptance of their abstracts, similar to the existing policy in regard to photography during poster sessions. It is considered professional etiquette to respect the presenter's wishes on data sharing.

In summary, tweeting during scientific meetings promotes discussion on topics of interest among those who attend either in person or virtually. It provides a way to find out about upcoming or ongoing popular sessions and helps expand professional networks through connecting with people whom we otherwise might not meet.

\section{REFERENCES}

1. Hawkins CM, Duszak R, Rawson JV. Social media in radiology: early trends in Twitter microblogging at radiology's largest international meeting. J Am Coll Radiol 2014;11:387-90

2. Wilkinson SE, Basto MY, Perovic G, et al. The social media revolution is changing the conference experience: analytics and trends from eight international meetings. BJU Int 2015;115:839-46

3. Neill A, Cronin JJ, Brannigan D, et al. The impact of social media on a major international emergency medicine conference. Emerg Med J 2014;31:401-04

4. Logghe $\mathrm{H}$, Maa J, Schwartz J. Twitter usage at Clinical Congress rises markedly over two years. Bull Am Coll Surg 2013;98:22-24

5. McKendrick DR, Cumming GP, Lee AJ. Increased use of Twitter at a medical conference: a report and a review of the educational opportunities. J Med Internet Res 2012;14:e176

6. Kolowich S. The academic twitterazzi. Technology. October 2, 2012. https://www.insidehighered.com/news/2012/10/02/scholars-debateetiquette-live-tweeting-academic-conferences. Accessed September 15,2014

7. Fitzgerald RT, Radmanesh A. Social media and research visibility. AJNR Am J Neuroradiol 2015;36:637

8. Radmanesh A, Fitzgerald RT. Social media and the neuroradiologist: a brief introduction. AJNR Am J Neuroradiol 2015;36:30-31

9. Croxall B. Ten tips for tweeting at conferences. The Chronicle of Higher Education. January 6, 2014. http://chronicle.com/blogs/ profhacker/ten-tips-for-tweeting-at-conferences/54281. Accessed September 14, 2014 